\title{
Development of a Risk Index for Use in Water Quality Monitoring
}

\author{
Lisa Jones $^{1} \cdot$ David Styles $^{1} \cdot$ Brian Kinsella $^{2} \cdot$ Antoin Lawlor $^{1} \cdot$ Ambrose Furey $^{2}$. \\ Fiona Regan ${ }^{1}$
}

Received: 24 August 2016 / Revised: 20 October 2016 / Accepted: 27 October 2016 / Published online: 29 November 2016

(C) Springer Science+Business Media Singapore 2016

\begin{abstract}
Risk indices have recently emerged as an effective and efficient tool in the area of water quality monitoring with new indices taking in vast quantities of data and facilitating the development of more targeted water monitoring programs. With the Water Framework Directive demanding that monitoring requirements for a list of priority substances be met, achieving 'good' status in all water bodies by 2015 , there is a strong need for improved monitoring programmes. In order to improve future monitoring programmes by making the process more 'targeted' a simple risk-based index for the occurrence of priority substances in wastewater treatment plant effluent was devised. This index was developed through the collection of an extensive list of documents relating to priority substance emission factors. These included wastewater treatment licence applications, trade effluent licences, traffic data, rainfall data, and census data. It was found that by relating data from each of these sources to historic occurrence data, it was possible to conceptualise and develop a ranking of risk of occurrence of priority substances. Validation of this index was carried out using data from a 24-month sampling plan at nine sites in two counties in Ireland representative of different pressures, i.e. agricultural, industrial and domestic. This work has allowed for the compilation of a large dataset of emission factor and priority substance occurrence in Ireland where none
\end{abstract}

Electronic supplementary material The online version of this article (doi:10.1007/s41101-016-0014-y) contains supplementary material, which is available to authorized users.

Fiona Regan

fiona.regan@dcu.ie

1 Water Institute, School of Chemical Sciences, Dublin City University, Glasnevin, Dublin 11, Ireland

2 Cork Institute of Technology, Cork, Ireland previously existed. For the first time, a risk-based index has been developed for Irish wastewater treatment plant effluents. Together, the index and dataset can be used by policy makers and inform the development of future priority substance monitoring programmes.

Keywords Risk · Water quality $\cdot$ Model $\cdot$ Water framework directive $\cdot$ Wastewater

\section{Introduction}

Priority substances (PSs) originate from many sources and are transferred to surface waters via a number of pathways. Large installations may emit PSs originating from production processes directly into surface waters under licence from the Environmental Protection Agency (EPA) or Local Authorities (LA). Gaseous emissions of PSs from combustion and industrial sources may be deposited on surrounding land and washed into surface waters directly following precipitation [1]. Accidental or deliberate dumping of waste materials onto land may also lead to run-off of PSs into surface waters, or indirect contamination of surface waters via leaching into groundwater [2].

Wastewater treatment plants (WWTPs) are potential major point sources of priority pollutants that combine direct inputs from domestic, industrial, and commercial effluent with diffuse inputs from surface run-off of land-deposited PSs. Sampling of PS concentrations in WWTP effluent under different conditions (e.g. wet and dry weathers) may offer insight into the sources of PS [3]. Furthermore, as major point sources of PSs and following implementation of the Urban Waste Water Treatment Directive [4], WWTPs offer strong opportunities for the effective control of PS concentrations in surface waters through the implementation of management and abatement options. Establishing the concentrations of priority 
pollutants in WWTP effluent is important for the protection of public health.

The EU Water Framework Directive (WFD) (2000/60/EC) established strict requirements for the monitoring of water quality in EU member states with a deadline of 2015, later pushed to 2021, for all water bodies to be of 'good' status. The levels of pollutants present in water bodies are most commonly judged against set environmental quality standards (EQSs) that vary among different countries. These standards dictate the maximum allowable concentrations (MAC EQS) or range of concentrations (annual average or AA EQS) of specific pollutants allowed to ensure compliance with the EC guidelines. Directive 2008/105/EC [5] and S.I. 272 of 2009 [6] define the latest EQS values for surface waters across Europe for priority pollutants, those being substances listed in Annex X of the WFD and later amendments. The EU WFD was transposed into Irish Law in 2003 [7], and as such, these EQS values now form the basis of priority substance water monitoring in Ireland.

In order for future monitoring programmes to meet the requirements established by the WFD, the gaps in current knowledge must be identified. Where information is lacking for accurate emission factor data for a priority substance or group of substances, this must be gathered in order to produce truly representative sampling results at the end of any monitoring campaigns. The aim of this study is to develop an environmental risk index based on a wealth of data collected to inform future-targeted monitoring programmes and thus enable Ireland to meet the WFD monitoring requirements.

\section{Review of Modelling Approaches for these Priority Substances}

When surveying current and completed work in this area, two large studies were identified as good examples of where modelling has facilitated the monitoring of WFD priority pollutants in the European arena. Using the basic concepts of modelling, a risk index can be devised. The SCOREPP [8] project (Source Control Options for Reducing Emissions of Priority Pollutants) had already surveyed a number of large European cities for the occurrence of priority substances and also included a section on removal efficiencies of specific pollutants from WWTPs that was particularly relevant to this study. Also, the Scottish Environmental Protection Agency (SEPA) has developed an efficient monitoring tool [9] and has reported the detailed process involved in establishing a model relating emission factors to the occurrence of priority substances in surface waters.

In order to determine the most suitable approach to adopt when preparing an index for the monitoring of emission factors for priority and hazardous substances in the Irish context, it was first necessary to evaluate some of the potential routes available. In a study by Ahlman and Svensson [10], a simple GIS-based model is presented, SEWSYS, which uses basic process parameters (deposition rates, accumulation time, flushing rates during rainfall) to predict storm water PS concentrations. It focuses on land use, including roof area and material type, road area and traffic volume. This model attributes most heavy metal and polycyclic aromatic hydrocarbon (PAH) loading to traffic (brake dust, tyre wear, exhaust and road surface wear). While this model provides valuable information on the type of data required and steps in establishing a basic working model, there were, however, many limitations which would have to be overcome. The SEWSYS model is based on the MATLAB program, computing software. The aim of the current study is to base the model in a simpler program such as Excel. Also compared to the large number of pollutants required for monitoring by the WFD, the study by Ahlman et al. contains only 20 different substances (some organic pollutants, heavy metals and nutrients). As a much larger pollutant set with more varying physicochemical characteristics and emission sources is being looked at in this study, the SEWSYS model, while being a good stepping stone, would ultimately need more work before being suitable for the purposes of this project. Further, while the hydrological aspects of the SEWSYS model were well validated, there were less reliable results in the quality parts.

De Keyser et al. [11] generated a dynamic model capable of simulating the dynamic release pattern of specific PSs. This model accounts for daily, weekly, and yearly patterns of release, but is extremely data intensive (geographic and average load data required for every source within a catchment), and the model was not tested on an actual catchment. Where sufficient data are available, high-resolution parameter-based models provide an invaluable tool to predict and understand temporal emission patterns and to interpret (grab sampling) monitoring data in the full context of dynamic relationships among various PS sources. However, in Ireland at least, the current state of knowledge and data availability (e.g. emission data from individual installations) is insufficient to support such models. In the first step towards greater understanding of PS sources contributing to WWTP emissions, a simple, stochastic, risk-based index that can be applied across catchments was proposed.

\section{Methodology}

\section{Design and Population of Index}

Data availability was a critical deciding factor in the design approach of the index which later impacted on the population of the index with available data. It was important to both recognise and minimise the limitations of the index from the beginning. In order to design an index that would allow for the 
determination of risk factors for priority substance emission in various catchment types, it was necessary to first identify all sources of background information for the population of this index. Figure 1 shows the relationship between the various data collected.

The design of the index involved a simple risk-based approach. This was the most effective way to demonstrate the need for targeted monitoring programs for priority substances. The risk rankings enable clear categorisation and ranking of various emission factors of priority substances according to the associated risk of loading attributed to each of those factors, Table 1 . The risk rankings are assigned based on collected data from a number of sources in conjunction with the experimental results of a sampling campaign at each of the sites included in the study. By relating the emission data to the actual determined levels in the wastewater effluent, it is possible to validate the risk rankings assigned within the index, or indeed re-evaluate an assigned rank.

For the purpose of this project, Microsoft Office Excel was chosen as the platform for this index due to its simple user interface, widespread availability and as a low-cost program that would be receptive to adequate volumes of data.

\section{Selecting the Agglomeration}

Nine sites in two counties were chosen on the basis that a spread of varying population equivalents, levels and types of treatment, and input to the treatment plants would best support a broad framework of knowledge from which to construct an index.

The counties of Dublin and Cork were chosen to best represent these requirements. These sites are summarised in
Table 1. Risk ranking scale applied to the data for the index

\begin{tabular}{ll}
\hline Risk ranking & Description (high possibility of...) \\
\hline 0 & No loading \\
1 & Light loading \\
2 & Significant loading \\
3 & Substantial loading \\
4 & Heavy loading \\
\hline
\end{tabular}

Table 2 below. The WWTPs are numbered 1-9 below with 1-7 located in Cork and 8-9 located in Dublin.

The flow chart in Fig. 2 details the first steps taken towards creating and populating the index. The variation in agglomeration provides the potential to test the results for any population size and WWTP source makeup.

To facilitate the preparation of a valuable tool, it was necessary to collect any available information on each site in order to build complete datasets. Included in the wastewater discharge licence applications for each of the treatment plants is an agglomeration map, a map of the catchment which shows the catchment area and any sub areas, and grid references for the plants are also included.

Using this information, it was possible to georeference each catchment map to a Google hybrid layer, the catchment borders are plotted, and conversions are carried out in order to relate map distances to actual areas. This was carried out using the QGIS mapping software.

In order to populate the index with relevant, high-quality data, it was necessary to evaluate the best possible sources of this data. Having characterised the respective agglomerations, an evaluation into the significant sources of priority

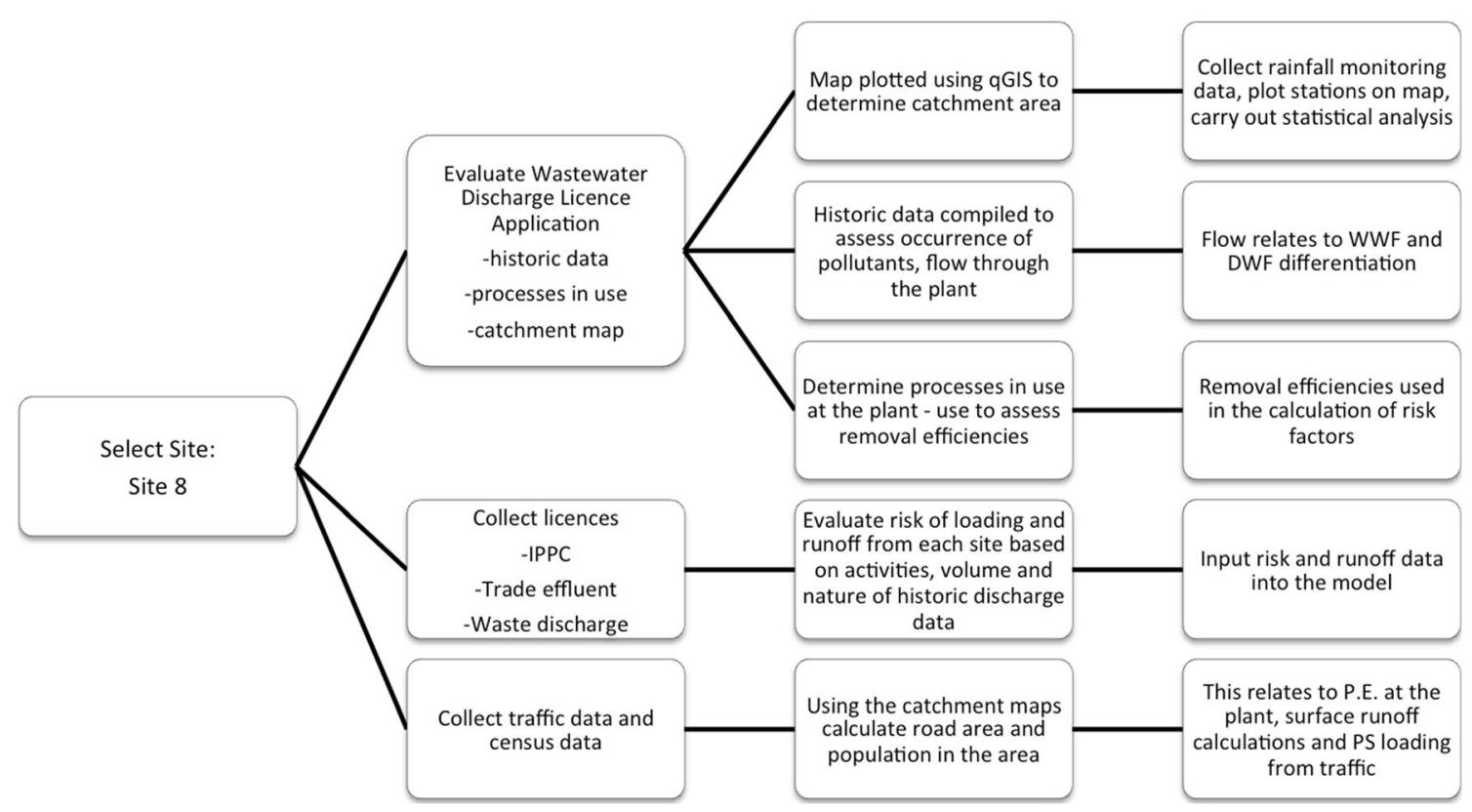

Fig. 1 Flow chart illustrating the steps taken in the development of the risk index, using Site 8 as an example 
Table 2. Overview of the WWTPs in this study. This information was gathered from the EPA wastewater licence applications of the respective WWTPs and from the EPA Urban Wastewater Report, 2007

\begin{tabular}{|c|c|c|c|c|c|c|}
\hline Site code & Treatment & Type of treatment & Agglom. PE & Plant PE & Area (Ha) & Receiving waters \\
\hline 1 & Secondary & PS, SS, AS (aeration basin) & 16,339 & 15,000 & 760 & Freshwater (R) \\
\hline 2 & Secondary & PS, AS (OD) & 8178 & 20,000 & 458 & Freshwater (R) \\
\hline 3 & Secondary & PS, SS, AS (OD) & 2984 & 6415 & 274 & Freshwater (R) \\
\hline 4 & Secondary & PS, SS, AS (OD) & $7500-15,000$ & 15,000 & 750 & Estuarine \\
\hline 5 & Secondary, NR & PS, SS, AS (OD; A-A-AT) PR & 5800 & 12,960 & 394 & Freshwater (R) \\
\hline 6 & Secondary, NR & PS, SS, AS (A-A-AT) PR & 7091 & 12,000 & 595 & Freshwater (R) \\
\hline 7 & None & None & 14,864 & 0 & 967 & Estuarine \\
\hline 8 & Tertiary & $\begin{array}{l}\text { PS, SS, AS (sequencing batch reactors) } \\
\text { U. V disinfection }\end{array}$ & $2,870,333$ & $1,640,000$ & 26,728 & Estuarine \\
\hline 9 & Secondary & PS, SS, AS (A-A-AT) & 50,000 & 60,000 & 2673 & Estuarine \\
\hline
\end{tabular}

Area area of catchment, $P E$ population equivalent, $R$ river, $P S$ primary settlement, $S S$ secondary settlement, $A S$ activated sludge, $P R$ phosphorous removal, $O D$ oxidation ditches, $A-A-A T$ anaerobic-, anoxic- and aeration tanks

substances in each, beginning with licenced emissions, was carried out. These emissions are listed in Fig. 2.

Industrial installations performing activities listed in the IPPC licence above specified thresholds are licenced under the EU Integrated Pollution Prevention and Control (IPPC) Directive. The Irish EPA makes licence conditions and Annual Environmental Reports (AER) on emissions from these installations publicly available. However, the level of information provided on PS emissions varies across installations and for the initial purposes of this risk-based index defined risk factors for each installation according to activity class were refined based on some installation-specific information provided in licences (whether or not there was discharge to sewers and surface water management). Sewer loadings of PSs from licenced installations were separated into direct sewer inputs and potential surface run-off inputs via

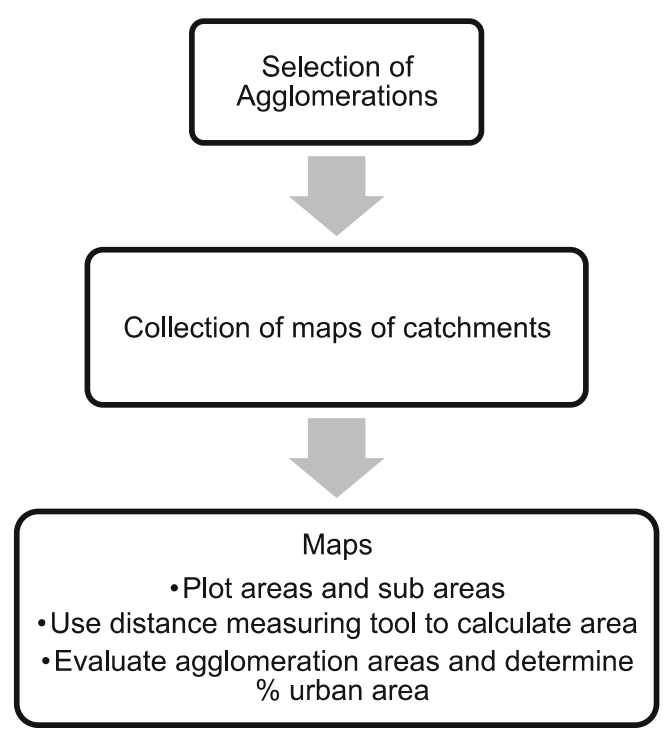

Fig. 2 Preliminary data obtained from agglomeration mapping combined drainage. The same simple risk index scheme was applied to each installation in relation to each PS group.

Waste management sites are also regulated by the EPA under Waste Licences. Local authorities issue Trade Effluent licences to commercial premises discharging to sewers. These licences typically contain little information; however, the type and number of each discharge was used in the loading calculations for each catchment. Lists of commercial installations were obtained from the relevant local authorities (excludes restaurants owing to low PS emission risk relative to domestic loading). Sites were categorised based on their primary activity (from internet searches where necessary), and loading risk factors were estimated for each PS group for each activity category.

In all cases, site addresses were cross-referenced with agglomeration maps in order to determine which, if any, WWTP agglomeration each sites feeds into. To convert risk factors into estimated loading factors for the risk model, consider loading is considered to be exponentially related to the four risk factors (risk factor 0 was not exponentially transformedit was left as 0 loading). This reflected the wide range of loading expected from different licenced sources with large IPPC sites, for example, discharging up to 100 times the volume of commercial sites, albeit with more stringently monitored controls. Some degree of emission abatement is in place for most licenced sites and emission loading will often depend on the effectiveness of the abatement methods (for run-off, this may depend on precipitation intensity).

It was found that not all businesses in the agglomeration applied for trade effluent licences. They exclude a larger proportion of businesses in the small agglomerations where sites may not be linked to the sewer network. Some such sites (e.g. garages) may still contribute to sewer PS loading via surface run-off (some of these are represented by surface run-off rankings attributed to waste licences in the database). Therefore, sewer loading of PSs from licenced installations was separated 
into direct sewer inputs and potential surface run-off inputs via combined drainage. This can also be a problem with other unlicensed sources such as car washes, Bed and Breakfasts, shops, pubs, sporting facilities, etc., which are not taken into account. For example, in 2008, it was reported that pollution loads arising from commercial sources were underestimated in the design at Ringsend WWTP, Dublin, and the same report estimated the actual contributed population equivalent from these commercial sources to be 190,000 on top of the pollution load arriving at Ringsend WWTP [12]. These sources are difficult to account for in this process, and this must be considered when establishing a water-monitoring programme.

\section{Wastewater Effluent Discharge Licence Applications and Annual Environmental Reports (AERs)}

While the maps were an important source of background information for the index, one of the greatest sources of large amounts of data required for populating the index was the wastewater effluent discharge licence applications and the AERs for each of the plants. Where available, these documents provided a wealth of information, as depicted in Fig. 3.

Three critical factors were derived from the available WWTP operational data to estimate the equivalent level of treatment achieved by each WWTP under dry weather flow (DWF) and wet weather flow (WWF) conditions:

- Level of treatment under normal operating conditions;

- DWF load factor;

- WWF load factor.

As well as grid reference values and sampling dates; the documents provided information on the population equivalent of the agglomeration. This was broken down into domestic and licenced contributions. This allowed characterisation of the loading factors to the plant to be carried out. Biological and hydraulic capacities of each plant were also included; however, the flow values were of the most value as these facilitated calculations of base flow through each plant allowing for the distinction of WWF and DWF conditions in relation to specific sampling results. The flow data also aided in the calculation of loading factors and was used to evaluate the performance of a specific plant in particular when it is operating above capacity. From the licence applications and AERs, a large dataset of historic sampling data was also compiled.

In order to assess PS loading, key risk indicators for both DWF and WWF conditions were devised. Table 3 shows the breakdown of each plant to show agglomeration population (taken from census records), population equivalent (P.E.) (this is the equivalent population of the area including both domestic and licenced inputs; this value is generally taken from the licence application except in the cases of WWTP8 and WWTP3 which were derived from estimated industrial flow BOD loading and adjustment using standard $16 \%$ estimate of industrial input, respectively), DWF levels and peak flow (PF) levels at pre-treatment (PF_PreT), at primary treatment $\left(\mathrm{PF} \_1 \mathrm{~T}\right)$ and at secondary treatment $(\mathrm{PF}$ 2T) stages, where the information was available. From the licence applications and AERs, the combined drainage area for each plant was also determined in broad 'mostly' or 'partly' terms; therefore, the assigned combined drainage (Comb Drain) factor values for each are shown below to be 0.75 for 'mostly' and 0.50 for 'partly', these factors were assigned taking into account the ratios of combined drainage from major inputs in the catchment.

For the Dublin sites, larger datasets were available and were provided by the plant operators. Files detailing flow, storm water discharge and storage levels, standard wastewater quality test results as well as some specific pollutant analysis results, specifically those for heavy metals where the data was available, were supplied. This data was not available for the Cork sites; therefore, licence application values were used as base levels and average levels in later calculations.

\section{EPA Waste Licences}

The EPA collects waste licence information, and this is made freely available on the Irish EPA website (www.epa.ie). All waste licences available for each agglomeration under study were collected; this enabled risk factors and loading factors for both direct input and run-off risk, Table 4 below, to be assigned based on the literature and available information.

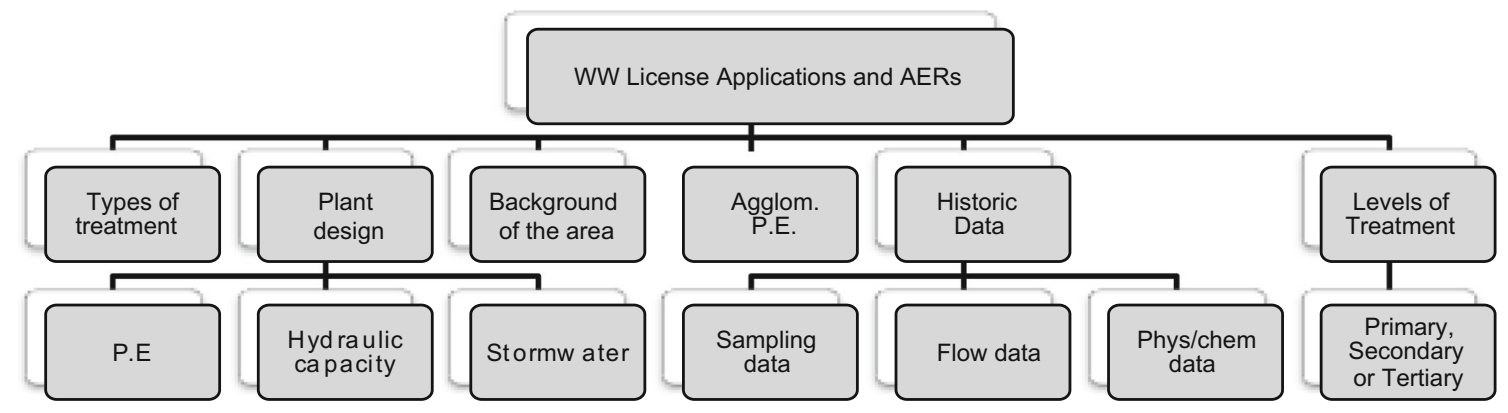

Fig. 3 Information included in the WWTP effluent discharge licence applications and annual environmental reports (AERs) 
Table 3 Spread sheet wherein the population, P.E., plant P.E., area of the catchment and flow data for each site were examined in order to determine peak flows (PF)

\begin{tabular}{|c|c|c|c|c|c|c|c|c|}
\hline Site & Level & Agglom pop. & Agglom PE & Plant PE & DWF & $\begin{array}{l}\text { PF 2T } \\
\mathrm{m}^{3} \text { day }^{-1}\end{array}$ & PF_1T & Comb. drain \\
\hline 1 & 2 & 16,339 & 24,524 & 26,000 & 2,700 & 12,216 & & 0.75 \\
\hline 2 & 2 & 6,200 & 8,178 & 20,000 & 2,160 & 6,480 & 13,478 & 0.75 \\
\hline 3 & 2 & 2,984 & 3,461 & 15,000 & 2,050 & 12,240 & 12,240 & 0.50 \\
\hline 4 & 2 & 11,250 & 13,050 & 6,067 & 1,469 & 8,813 & 8,813 & 0.50 \\
\hline 5 & $2, \mathrm{NR}$ & 5,800 & 12,000 & 20,000 & 3,200 & 16,152 & & 0.50 \\
\hline 6 & $2, \mathrm{NR}$ & 7,091 & 8,226 & 18,000 & 5,338 & 13,344 & & 0.50 \\
\hline 7 & 0 & 14,864 & 97,556 & 97,556 & 9,000 & N/A & N/A & 0.50 \\
\hline 8 & 3 & $1,200,000$ & $1,708,000$ & $1,640,000$ & 377,568 & 959,040 & 911,088 & 0.50 \\
\hline 9 & $2, \mathrm{NR}$ & 50,000 & 58,000 & 60,000 & 12,063 & & $100 \%$ & 0.75 \\
\hline
\end{tabular}

Level level of treatment at the plant i.e. primary(1), secondary(2), tertiary(3) and none(0), NR nutrient removal, Comb. drain combined drainage in the catchment, $N / A$ not applicable

Taking a closer look at the EPA waste licence applied for by the Cork County Council for a landfill facility, which feeds into WWTP2, Table 4 below, it can be seen that risk factors for all PS from direct input were assigned a value of 4 , while runoff risk was assigned 0 . These values were determined from evaluation of the waste licence application and accompanying documents.

Information on the nature of the facility, the quantity and nature of the waste, plant methods and operating procedures, nature and impact of emissions, results of monitoring and sampling, the recovery and treatment of waste and unauthorised of unexpected emissions was also available. This data was verified through comparison to the latest AER which provides further information on current waste quantities and composition, site capacity, any works at the site,

Table 4 Examples of some EPA waste licence holders, the WWTP they contribute to risk factors and loading factors for both direct input and runoff risks

\begin{tabular}{|c|c|c|c|c|c|c|c|c|c|}
\hline \multirow[t]{3}{*}{ Code } & \multirow[t]{3}{*}{ Plant } & \multicolumn{8}{|c|}{ Risk factor } \\
\hline & & \multicolumn{4}{|c|}{ Direct input } & \multicolumn{4}{|c|}{ Run-off risk } \\
\hline & & РAH & VOC & $\mathrm{HM}$ & Pest. & $\mathrm{PAH}$ & VOC & HM & Pest. \\
\hline LF & 2 & 4 & 4 & 4 & 4 & 0 & 0 & 0 & 0 \\
\hline WTF & 4 & 2 & 2 & 2 & 1 & 2 & 2 & 2 & 2 \\
\hline HW & 5 & 3 & 3 & 3 & 3 & 0 & 0 & 0 & 0 \\
\hline \multirow[t]{4}{*}{ IWM } & 8 & 2 & 2 & 2 & 1 & 2 & 2 & 2 & 2 \\
\hline & & \multicolumn{8}{|c|}{ Loading factor } \\
\hline & & \multicolumn{4}{|c|}{ Direct input } & \multicolumn{4}{|c|}{ Run-off risk } \\
\hline & & PAH & VOC & HM & Pest. & $\mathrm{PAH}$ & VOC & HM & Pest. \\
\hline LF & 2 & 54.6 & 54.6 & 54.6 & 54.6 & 0 & 0 & 0 & 0 \\
\hline WTF & 4 & 7.4 & 7.4 & 7.4 & 2.7 & 7.4 & 7.4 & 7.4 & 7.4 \\
\hline HW & 5 & 20.1 & 20.1 & 20.1 & 20.1 & 0 & 0 & 0 & 0 \\
\hline IWM & 8 & 7.4 & 7.4 & 7.4 & 2.7 & 7.4 & 7.4 & 7.4 & 7.4 \\
\hline
\end{tabular}

$L F$ landfill, $W T F$ waste transfer station, $H W$ hazardous waste facility, $I W M$ integrated waste management facility continuous monitoring systems and sampling results and any environmental incidents or non-compliances.

This data was gathered and evaluated for each of the licenced waste facilities within each of the catchments in this study; however, there is always the issue of illegal dumping which is difficult to take into account; therefore, for the purposes of this study, only licenced sources could be included.

\section{IPPC Licences}

As with the EPA waste licences, the IPPC licences for each catchment were gathered and evaluated as shown in Table 5 . Licence applications and accompanying documents were reviewed. Risk and loading factors for direct input and runoff risk were attributed to each of the industries.

Table 5 Examples of some IPPC licence holders, their addresses, and risk factors and loading factors for both direct input and run-off risks

Code Plant Risk factor

Direct input Run-off risk

PAH VOC HM Pest. PAH VOC HM Pest.

$\begin{array}{llllllllll}\mathrm{CL} & 8 & 2 & 4 & 2 & 2 & 1 & 1 & 1 & 0\end{array}$

$\begin{array}{llllllllll}\mathrm{FD} & 2 & 0 & 0 & 0 & 0 & 1 & 1 & 1 & 0\end{array}$

$\begin{array}{llllllllll}\mathrm{M} & 3 & 2 & 2 & 4 & 0 & 1 & 1 & 1 & 0\end{array}$

$\begin{array}{llllllllll}\mathrm{OR} & 6 & 1 & 3 & 3 & 0 & 1 & 1 & 1 & 0\end{array}$

Loading factor

Direct input Direct input

PAH VOC HM Pest. PAH VOC HM Pest.

$\begin{array}{llllllllll}\mathrm{CL} & 8 & 7.4 & 54.6 & 7.4 & 7.4 & 2.7 & 2.7 & 2.7 & 0\end{array}$

$\begin{array}{llllllllll}\text { FD } & 2 & 0 & 0 & 0 & 0 & 2.7 & 2.7 & 2.7 & 0\end{array}$

$\begin{array}{llllllllll}\mathrm{M} & 3 & 7.4 & 7.4 & 54.6 & 0 & 2.7 & 2.7 & 2.7 & 0\end{array}$

$\begin{array}{llllllllll}\text { OR } & 6 & 2.7 & 20.1 & 20.1 & 0 & 7.4 & 7.4 & 7.4 & 7.4\end{array}$

$C L$ chemical industry, $F D$ food and drink facility, $M$ metals facility, $O R$ other activities 
In the most recent AER (2010) for this site (WWTP7), the only listed emissions to water/sewers are rainwater to the field drain, clean water to the field drain (emptying clean tanks filled with water for pressure testing) and office and canteen toilets connected to the mains sewerage system (volumes not available). Descriptions and quantities of disposed waste are also detailed in this report, and as such, this catchment was risk ranked for direct input of PAHs, volatile organic compounds (VOCs) and heavy metals (HMs).

\section{Trade Effluent Licences}

Loading data and trade effluent licences were acquired for each agglomeration under study. The licence information provided a broad outline of industrial and commercial inputs of specific PSs to the surface waters in the agglomeration based on the licenced activities.

Lists of commercial installations were obtained from the relevant local authorities (this excludes restaurants which are licenced under separate fats, oils and greases licences and were excluded from this study owing to low PS emission risk relative to domestic loading). Sites were categorised based on their primary activity, and loading risk factors were estimated for each PS group for each activity category.

\section{Rainfall Data}

Rainfall creates a flushing effect at the WWTP as this rainwater brings with it many pollutants that had been present as street dust, pesticides on grass and fields, and airborne particulates. Increased flow reaching the plant often pushes a plant above capacity, and the stream is diverted to storm water overflow where the water can be released, untreated, back into the effluent stream. Met Éireann provided an extensive list of rainfall monitoring stations nationwide, including grid references which allowed for their mapping.

This data was used to assess the dry periods, rain intensity and rain duration and the effect of meteorological conditions at the time of sampling. It is important to emphasise that the index simply ranks risk relative to average agglomerationsfor dry - and wet weather flow. Further work is required to define meteorological relationships for more detailed risk rankings in particular to account for the preceding dry period.

After plotting the rainfall stations, determination of the sites which would give the most accurate representation of the level of rainfall reaching the WWTPs was conducted. A study was carried out to discover which stations had the most representative values in relation to the site. Sites within a $5 \mathrm{Km}$ radius of each of the sites were selected , and statistical analysis was carried out to determine the levels of correlation between the rainfalls measured at each of the sites. This was determined using the Pearson correlation coefficient. It was then possible to take the mean rainfall of the monitoring stations when comparing to the flow through the plant, and, in turn, the detected pollutant levels.

Where detailed flow data was available, it was possible to establish base flow levels and assign benchmarks above and below which dry and wet weather flow conditions could be defined. Otherwise, flow data provided in the licence applications was used. The flow data was evaluated in order to determine a base level flow through the plant in relation to rainfall conditions at the time; this allowed for the definition of WWF and DWF parameters for the Cork sites. For the final index, the separation of WWF and DWF results was necessary; therefore, this was an important step.

\section{Traffic Data}

Traffic data obtained from the National Roads Authority (NRA) as well as the Central Statistics Office (CSO) was used in conjunction with mapping techniques that allowed for the determination of average road usage in each catchment. Traffic-induced loading to WWTP (PAH, VOCs, HMs) should be largely proportional to traffic volume within agglomeration combined drainage areas, as measured by Vehicle Km Travelled (VKT). Relevant statistics included traffic flow on national roads, total VKT for different vehicle types, [13], and national VKT for major road types, [14].

Traffic was divided into two components: local traffic and through traffic. Local traffic was considered to be a function of agglomeration population and was calculated based on the national average VKT per car on regional and local roads estimated to occur within urbanised (i.e. WWTP agglomeration) areas - it was assumed that national average car ownership data ([14]) translated into a ratio of 0.4 cars per capita in Dublin city and 0.5 cars per capita elsewhere in Ireland (Table 6).

Local VKT within agglomerations was taken to equal the standard VKT per car (2359 $\mathrm{Km} \mathrm{a}^{-1}, 2949 \mathrm{Km} \mathrm{a}^{-1}$ in Dublin), or twice the agglomeration length if this was smaller (to bound traffic volume by catchment size for smaller catchments). Local Heavy Commercial Vehicle (HCV) traffic (lorries and buses) was calculated as a fixed ratio to car traffic based on the CSO (2009) data. A weighting factor of 3 was applied to HCV traffic to generate car equivalent VKMs that reflect higher PS deposition from HCVs (e.g. greater road wear and generation of brake dust, higher fuel use.) The estimated proportional area under combined drainage was multiplied by agglomeration VKT to estimate the traffic-loading index for the catchment.

Through traffic was assumed to occur on national roads ( N roads) within agglomeration boundaries. National road traffic count data for points on $\mathrm{N}$ roads in the vicinity of agglomerations (NRA, 2009) were multiplied by the length of the $\mathrm{N}$ road within agglomeration boundaries to generate $\mathrm{N}$ road VKT for each agglomeration. $\mathrm{N}$ roads were classified as 
Table 6 Summary table showing traffic data gathered for each of the catchments in the study. Each catchment is broken down into population, local traffic, traffic distributions, a combined drainage estimate, types of vehicle, equivalent vehicles per kilometre travelled (eq VKT) and an overall ranking of traffic loads for each WWTP in order from most (1) to least (10)

\begin{tabular}{|c|c|c|c|c|c|c|}
\hline \multirow[t]{2}{*}{ Site } & \multicolumn{3}{|l|}{ Cars } & \multicolumn{3}{|l|}{ Heavy commercial vehicles } \\
\hline & $\begin{array}{c}\text { Local traffic } \\
\text { corrected }\end{array}$ & N Rd. traffic-C & N-Rd. traffic-P & Local traffic & N Rd. traffic-C & N Rd. traffic-P \\
\hline 1 & $19,275,015$ & & $16,811,941$ & $7,100,215$ & & 605,230 \\
\hline 2 & $5,095,151$ & $18,294,390$ & & $1,876,868$ & $1,324,682$ & \\
\hline 3 & $2,399,192$ & $27,557,100$ & & 883,775 & $3,058,838$ & \\
\hline 4 & $5,134,615$ & & $11,325,335$ & $1,891,406$ & & $1,018,271$ \\
\hline 5 & $4,199,673$ & $4,832,484$ & $3,487,179$ & $1,547,007$ & 401,247 & 484,718 \\
\hline 6 & $6,847,523$ & $2,089,568$ & $23,298,954$ & $2,522,378$ & 156,718 & $2,073,607$ \\
\hline 7 & $17,534,967$ & & $9,990,981$ & $6,459,245$ & & $1,768,952$ \\
\hline 8 & $1,415,632,449$ & & $956,110,597$ & $521,467,533$ & & $96,861,695$ \\
\hline 9 & $58,984,685$ & & $85,061,453$ & $21,727,814$ & & $6,124,425$ \\
\hline & Pop. & Comb. sewer & Relevant car eq VKT & Add car eq VKT & Car eq vKm C/C. A & Rank \\
\hline & & & Central and combined sewer & $\begin{array}{l}\text { Peripheral and central } \\
\text { non-combined }\end{array}$ & & \\
\hline 1 & 16,339 & 0.75 & $30,431,745$ & $28,771,546$ & 53,404 & 5 \\
\hline 2 & 6200 & 0.75 & $24,745,644$ & $8,248,548$ & 72,087 & 3 \\
\hline 3 & 2964 & 0.50 & $20,892,065$ & $20,892,065$ & 152,506 & 1 \\
\hline 4 & 11,250 & 0.50 & $5,404,416$ & $19,784,564$ & 14,415 & 10 \\
\hline 5 & 5800 & 0.50 & $7,438,458$ & $12,379,790$ & 37,803 & 7 \\
\hline 6 & 7091 & 0.50 & $8,487,189$ & $38,006,963$ & 28,530 & 8 \\
\hline 7 & 14,854 & 0.50 & $27,684,526$ & $24,526,012$ & 57,238 & 4 \\
\hline 8 & $1,200,000$ & 0.50 & $1,490,017,524$ & $2,736,713,206$ & 111,496 & 2 \\
\hline 9 & 50,000 & 0.75 & $93,126,095$ & $134,476,758$ & 46,447 & 6 \\
\hline
\end{tabular}

$C$ central, $P$ peripheral

peripheral to the agglomeration (e.g. bypass roads) and central to the agglomeration. Peripheral roads were assumed to have independent drainage systems, whilst central roads were assumed to have combined drainage according to individual agglomeration proportional combined drainage estimates. For Dublin, where $\mathrm{N}$ roads radiate out of the centre and carry most commuter traffic to and from the city, through traffic was assumed equal to traffic on radial M50 motorway. NRA data is reported as the Annual Average Daily Traffic (AADT) and $\% \mathrm{HCV}$, enabling car-eq. VKT is to be calculated for each $\mathrm{N}$ road.

\section{WWTP Removal Efficiencies}

WWTPs can be major point source inputs of priority substances to surface waters, and while many studies show that wastewater treatment removes $90-95 \%$ of pollutants, this removal efficiency (R.E.) depends on a number of factors. In order to calculate the loading of PSs attributed to the WWTPs, it was necessary to determine approximate R.E.s for each of the pollutants within the respective plants.

This R.E. is directly related to the operations at the plant, the input to the plant, the treatment levels/types received by the water at each site, the handling of storm water and extreme weather events and the ScorePP project, Seriki et al. (2008) reported on measured PS removal efficiencies at WWTPs, mainly based on secondary treatment with activated sludge. For all WWTPs considered in this study, removal efficiencies for each group of PSs were approximated to average removal efficiencies for relevant PSs reported in [15].

After determining R.E.s for the priority substances, it was possible to compile all data collected (licenced information, rainfall and traffic data and removal data) into a functional index. The data was organised and tabulated as has been shown in such a way that excel functions could be applied between documents, forming a link and allowing for easy updating or alteration of data.

\section{Results}

\section{Conceptual Indexing}

Following the compilation of all relevant available data, the conceptual index was developed. This is the basis of the framework of the index and affords certain flexibility to the 
index with regards to additions to the background data. Many calculations were derived as part of the development of this index and are discussed and rationalised below.

WWTP load factors were calculated as a ratio of agglomeration PE loading to WWTP PE capacity. For WWTP4, with a calculated load factor of 2.2, DWF removal factors were based on the assumption that half the flow received secondary treatment and half received primary treatment only. For WWTPs working in excess of or close to capacity, WW removal factors were based on the assumption that overall removal efficiencies under high loading conditions were equivalent to primary treatment removal efficiencies. Removal factors were inversed into effluent factors (EF) for direct multiplication with loading factors.

The conceptual indexing of PS loading is to devise key risk indicators applicable under dry weather flow (DWF) and wet weather flow (WWF) conditions. To convert risk factors into estimated loading factors for the risk index, loading was considered to be exponentially related to the four risk factors. This reflects the wide range of loading expected from different licenced sources, with large IPPC sites for example discharging up to 100 times the volume of commercial sites.

Loading to the environment from each WWTP under DWF is expressed as DWF domestic loading (population size) plus DWF industrial loading (total agglomeration PE), multiplied by EFDWF (effluent factor for dry weather flow).

Under WWF, WWTP loading is a function of DWF loading, plus WWF Domestic loading, plus WWF Industrial loading, plus WWF traffic loading, plus WWF land use loading, all multiplied by EFWWF. It is assumed that industry and transport sources are major contributors to WWTP PS loading.

Using the concepts above which both collected data and the results of a 3-year sampling campaign, it was possible to produce a final index for the four main groups of priority substances (pesticides, PAHs, HMs and VOCs).

\section{Assigning Risk}

Assigning risk values for different PSs under different conditions follows a specific procedure and is used along with the conceptual indexing described above. For example, assigning risk factors based on IPPC licencing for a single catchment was carried out as outlined in Fig. 4.

This 4-step process enabled a structured methodology to assigning risk factors.

Using another example to illustrate assigning of risk values to the different catchments, the site of Site 1 is expanded upon below.

For example, for a single petrol station in this catchment, the risk of PAH, VOC and metals and trace elements occurrence is allocated a risk of 2 (per group) while pesticides are risked 0 . When all trade effluent licences for Site 1 have been

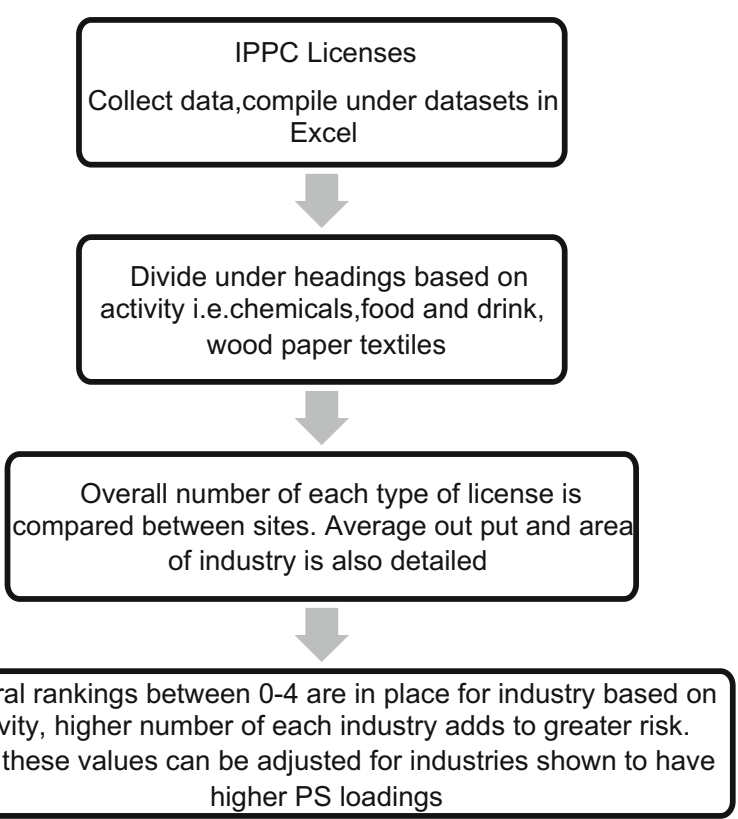

Fig. 4 Overview of procedures involved in assigning risk factors using IPPC licences as a sample source from single catchment

evaluated in this same manner, the same procedure is carried out for waste discharge licences and IPPC licences. As Site 1 had no licences other than trade effluent, this was the simplest site to index. The SUM risk for each group of pollutants assigned from the seven trade effluent licences was used to calculate final risk. For example, the SUM PAH risk for Site 1 was 38, Table 7 shows how this number relates to the final DWF risk for PAHs in Site 1.

Taking Site 1 as an example, and focussing on PAHs during DWF, Table 7 sets out how the final risk factor is achieved.

- The P.E. has been gathered from WWTP licence application and census records for this catchment.

- The calculation of the industrial risk factor has been outlined above in Fig. 5.

- The effluent factor is assigned as 0.2. This was derived from the standard removal efficiency of PAHs from secondary treatment. The effluent factor can be any value from 1 to 0 with 1 indicating no removal of the pollutant and 0 indicating $100 \%$ removal of the pollutant. The value of 0.2 here indicates $80 \%$ removal of PAHs at this site under normal operating conditions.

- The national population is under National P.E.

- The national industrial risk factor is the total number of trade effluent licences.

- The DWF risk is calculated.

- DWF Risk for PAHs = (Industrial risk divided by national industrial risk) divided by (P.E. divided by national P.E.) multiplied by the effluent factor $=0.09$ 
Table 7 Final calculation of DWF risk factor for PAHs in Site 1

\begin{tabular}{lrcll}
\hline & P.E. & Industrial risk factor & Effluent factor & DWF risk \\
\hline Site 1 & 17,989 & 38 & 0.2 & 0.09 \\
National & $4,240,000$ & 20,000 & & \\
\hline
\end{tabular}

\section{Model Validation}

The final step in the establishment of the risk index is the population of the index with analytical data from samples collected. This includes data collected at the 9 WWTPs over the 24-month (2009-2011) period for an overall sample set of 492 samples. Samples were analysed for the presence of trace levels of 8 PAHs, 14 pesticides and 15 metals and trace elements, with the VOC index based solely on collected historic data. Historic sampling datasets allow models to be populated

Fig. 5 Outline on assigning risk based on IPPC licences in WWTP1

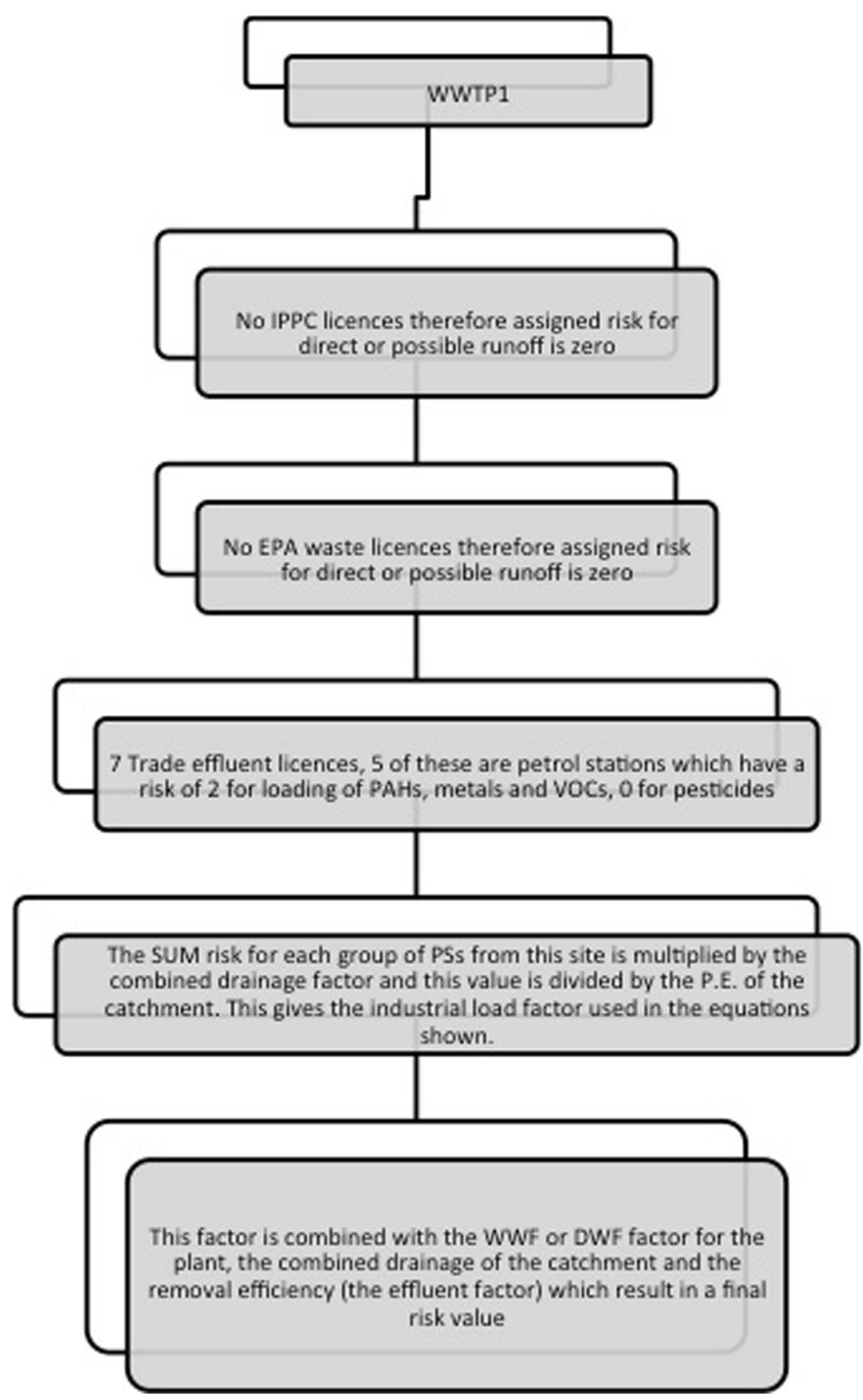


Table 8 Exceedances of priority substances at two WWTPs

\begin{tabular}{llc}
\hline & Site 1 & Site 8 \\
\hline PAHs & $\begin{array}{c}\text { Benzo-b/k-fluoranthene and } \\
\text { indeno-1,2,3 cd-pyrene/benzo-- } \\
\text { ghi-perylene all exceed EQS in 90 } \\
\text { percentile. }\end{array}$ & $\begin{array}{c}\text { Indeno-1,2,3 cd-pyrene/benzo-ghi-peryle- } \\
\text { ne exceed EQS above 90 percentile. }\end{array}$ \\
& Chlorfenvinphos and DEHP exceed & \\
EQS in 50 percentile. & $\begin{array}{c}\text { Alachlor, DEHP, chlorfenvinphos, diuron, } \\
\text { and chlorpyrifos exceed EQS in 90 } \\
\text { percentile. }\end{array}$ \\
Metals and & Chromium, copper, zinc and tin exceed \\
trace elements & Tin and lead exceed EQS above 50 & EQS in 50 percentile. \\
& percentile. & Nickel exceeds EQS in 90 percentile. \\
\hline
\end{tabular}

with high-quality, representative data, making the final index more reliable and robust. Through the sampling regime carried out in this project, it is important to highlight the value of intensive sampling data (Table 8).

Table 9 presents an overview of the final risk factors for each group of priority substances at the nine WWTPs under both wet and dry weather flow conditions. For the pesticides, VOCs and metals, it was found that there are consistently higher risks associated with wet weather flow conditions. This is representative of the higher run-off risk loadings attributed to sources of PSs in the catchments. The PAHs were found to have higher risk of direct loading from licenced sources than from run-off with traffic proving to be one of the largest contributors of PAHs. This would indicate that these sites should be monitored specifically for PAHs at periods of DWF when there is a greater likelihood of elevated priority substance occurrence to be detected.

Site 7 was found to be a high-risk site in all areas; this is owing to both contributions from licenced sources in the area and the lack of any treatment processes. This site acts as a pumping station only. By examining two of the sites more closely, Site 1 in Cork and Site 8 in Dublin, it is possible to validate the index making it applicable to any site.
These sites were chosen to be included in this index validation step as they represent both counties in the study, two different levels of treatment and removal efficiency and two different types of input to the respective plants, and were the sites with the most available data for collection and population of the index. They are representative of the range of conditions evaluated in the index. Specific flow data for each of these WWTPs allowed for a clear distinction between WWF and DWF conditions whereas a number of the other sites did not collect flow data sets, and flow data was determined solely from weather conditions and historic data.

A true comparison of the two sites can be demonstrated in Table 8 above. This table shows detected exceedence of EQS values for specific pollutants from each group of pollutants by site. The percentile of exceedence is also included to show the frequency of occurrence of these high levels of PSs.

Site 1 was found to have low risk attributed to all areas even during WWF while Site 8 is classified as at risk of high levels of both pesticides and metals under the same conditions. Using the heavy metals as an example, results can be rationalised as the metals and trace elements risk assessments met expectations with Site 8 ranked as a higher risk than Site 1. Site 8 shows both higher percentage frequency of

Table 9 Comparison of predicted risk to actual risk determined from sampling data for two sites

\begin{tabular}{|c|c|c|c|c|c|}
\hline & & \multicolumn{2}{|l|}{ Site 1} & \multicolumn{2}{|l|}{ Site 8} \\
\hline & & Predicted & Actual & Predicted & Actual \\
\hline \multirow[t]{2}{*}{ PAHs } & DWF & $\begin{array}{l}\text { Low risk } \\
(0.1)\end{array}$ & $\begin{array}{l}\text { Low risk } \\
(0.09)\end{array}$ & $\begin{array}{l}\text { Some risk } \\
(0.14)\end{array}$ & $\begin{array}{l}\text { Low risk } \\
(0.06)\end{array}$ \\
\hline & WWF & $\begin{array}{l}\text { Medium risk } \\
(0.15)\end{array}$ & $\begin{array}{l}\text { Medium risk } \\
(0.15)\end{array}$ & $\begin{array}{l}\text { Medium risk } \\
(0.15)\end{array}$ & $\begin{array}{l}\text { Medium risk } \\
(0.21)\end{array}$ \\
\hline \multirow[t]{2}{*}{ Pesticides } & DWF & $\begin{array}{l}\text { Low risk } \\
(0.02)\end{array}$ & $\begin{array}{l}\text { Low risk } \\
(0.02)\end{array}$ & $\begin{array}{l}\text { Some risk } \\
(0.08)\end{array}$ & $\begin{array}{l}\text { Low risk } \\
(0.03)\end{array}$ \\
\hline & WWF & $\begin{array}{l}\text { Low risk } \\
\text { (0) }\end{array}$ & $\begin{array}{l}\text { Low risk } \\
(0.04)\end{array}$ & $\begin{array}{l}\text { High/very high risk } \\
\text { (1.4) }\end{array}$ & $\begin{array}{l}\text { High/very high risk } \\
\text { (1.3) }\end{array}$ \\
\hline \multirow[t]{2}{*}{ Metals and trace elements } & DWF & $\begin{array}{l}\text { Low risk } \\
(0.05)\end{array}$ & $\begin{array}{l}\text { Low risk } \\
(0.05)\end{array}$ & $\begin{array}{l}\text { Low/some risk } \\
(0.08)\end{array}$ & $\begin{array}{l}\text { Low risk } \\
(0.04)\end{array}$ \\
\hline & WWF & $\begin{array}{l}\text { Some/medium risk } \\
(0.7)\end{array}$ & $\begin{array}{l}\text { Some/medium risk } \\
(0.66)\end{array}$ & $\begin{array}{l}\text { Medium/high risk } \\
(1.2)\end{array}$ & $\begin{array}{l}\text { Very high risk } \\
\text { (1.72) }\end{array}$ \\
\hline
\end{tabular}


Table 10 Final risk rankings attributed to each site for the main groups of WFD priority substances under both WWF and DWF conditions

\begin{tabular}{|c|c|c|c|c|c|c|}
\hline \multirow[t]{2}{*}{ Site } & \multicolumn{2}{|l|}{ PAHs } & \multicolumn{2}{|c|}{ Pesticides } & \multicolumn{2}{|c|}{ Metals } \\
\hline & DWF & WWF & DWF & WWF & DWF & WWF \\
\hline 1 & 0.09 & 0.15 & 0.02 & 0.04 & 0.05 & 0.66 \\
\hline 2 & 0.80 & 0.37 & 0.65 & 1.31 & 0.44 & 1.17 \\
\hline 3 & 1.90 & 0.09 & 1.75 & 3.50 & 1.39 & 2.32 \\
\hline 4 & 0.26 & 0.19 & 0.31 & 2.64 & 0.11 & 1.06 \\
\hline 5 & 0.48 & 0.05 & 0.38 & 0.77 & 0.26 & 0.69 \\
\hline 6 & 0.35 & 0.06 & 0.30 & 0.59 & 0.27 & 0.53 \\
\hline 7 & 3.79 & 0.82 & 1.60 & 7.10 & 1.44 & 5.55 \\
\hline 8 & 0.06 & 0.21 & 0.03 & 1.30 & 0.04 & 1.72 \\
\hline 9 & 0.06 & 0.03 & 0.01 & 0.08 & 0.04 & 0.29 \\
\hline
\end{tabular}

occurrence for the metals and trace elements, but also a wider variety of these substances than Site 1.

Table 8 shows results in agreement with the index with a number of metals found to exceed EQS levels at a higher percentage occurrence in Site 8 than for Site 1. Site 8 also covers a much larger catchment with a higher percentage urban area than site 1 (75\% compared to $70 \%)$. This catchment included 36 waste licences, 66 IPPC licences and 253 trade effluent licences, many of which are for laboratories, construction and machinery companies, the transport industry and other sources which are highly ranked for loading of PSs.

Through the comparison of these sites, the index has been found to be true to analytical results from a strict sampling regime with results fitting the index created based on a large dataset of licences, annual environmental reports and historic data. The index can be further adapted and is receptive to unlimited amounts of data making it amenable to continuous updating.

\section{Application of Index}

The main application of this index is the use in identifying the risks of priority substance occurrence at a site under both wet and dry weather conditions. This simple index can therefore be used in the development of future monitoring programmes, reducing the need for and cost of non-specific monitoring methods. Table 10 shows the final risk-based model for the occurrence of priority substances in wastewater. The rankings applied relate to the scale of risk of occurrence of priority substances, presented in Table 11. Standard font indicates a low risk of PS loading during certain conditions with risk progressively increasing to a high risk of loading indicated in bold. Site 7 has been ranked as very high risk owing to the lack of treatment of the wastewater at this site.

Through the collection of data, it can be concluded that the same ranking system can be applied to other catchments and can be used as a guidance document for the monitoring of PSs in an area. By generating risk factors using the methods described, the scale outlined in Table 11 below can be used to evaluate the risks of occurrence of PSs at a site under both wet and dry weather conditions.

\section{Conclusions}

This design and testing of a risk-based model for PS monitoring has been described. This work leads to the recommendation that standardised methods, and practices are required in order for any monitoring program to be a success. It is indices or tools such as this one which makes these monitoring programs possible, by informing the monitoring approach and highlighting the areas that require the most attention.

Although presented as a simple risk-based index relating emission factors to the occurrence of priority substances in wastewater, this index can be used to inform current monitoring, specifically highlighting current gaps in knowledge and recommending changes in current methods and practices.

As one of the main challenges encountered in the development of this index was the lack of data specific to indexing purposes; it is important to note these gaps in knowledge and to, where possible, strive to fill these gaps and maintain a level of data quality and availability which would facilitate future monitoring programs. Information must be collected on weather conditions, plant conditions and operations, and
Table 11 Ranking system converting risk factors to scale of risk of PS occurrence under different conditions

\begin{tabular}{|c|c|c|c|c|c|c|}
\hline \multirow[t]{2}{*}{ Scale } & \multicolumn{2}{|l|}{ PAHs } & \multicolumn{2}{|l|}{ Pesticides } & \multicolumn{2}{|l|}{ Metals } \\
\hline & DWF & WWF & DWF & WWF & DWF & WWF \\
\hline$<0.05$ & LR & LR & LR & LR & LR & LR \\
\hline $0.06-0.1$ & LR & LR-SR & SR & LR & LR-SR & LR \\
\hline $0.11-0.5$ & SR-MR & MR & MR & LR & SR-HR & LR-SR \\
\hline $0.51-1$ & MR-HR & HR-VHR & HR & SR-MR & HR-VHR & SR-MR \\
\hline $1.1-1.5$ & HR & VHR & HR-VHR & MR-HR & VHR & MR-HR \\
\hline$>1.5$ & VHR & VHR & VHR & HR-VHR & VHR & HR-VHR \\
\hline
\end{tabular}

$L R$ low risk, $S R$ some risk, $M R$ moderate risk, $H R$ high risk, $V H R$ very high risk 
unlicensed sources must be properly accounted for as they have been found to yield significant contributions of priority substances to wastewater and are still present in the WW discharge being released back to the environment. The availability of information on trade effluent, licenced effluent, and most importantly unlicensed sources, as well as usage information would allow for the future establishment of comprehensive monitoring programs which would enable Ireland to meet WFD requirements and EU targets in the area of water quality.

Acknowledgements The authors would like to acknowledge the Irish Environmental Protection Agency for funding this research and to the team members in Cork IT for their contributions.

\section{References}

1. Sanders G, Jones KC, Hamilton-Taylor J (1993) A simple method to assess the susceptibility of polynuclear aromatic hydrocarbons to photolytic decomposition. Atmos Environ Part A 27:139-144

2. Teijon G, Candela L, Tamoh K, Molina-Díaz A, Fernández-Alba A (2010) Occurrence of emerging contaminants, priority substances $(2008 / 105 / C E)$ and heavy metals in treated wastewater and groundwater at Depurbaix facility (Barcelona, Spain). Sci Total Environ 408:3584-3595
3. Gasperi J, Garnaud S, Rocher V, Moilleron R (2008) Priority pollutants in wastewater and combined sewer overflow. SciTotal Environ 407:263-272

4. EEC (1991) Council Directive of 21 May 1991 Concerning Urban Waste Water Treatment (91/271/EEC)

5. European Parliament (2008) Directive 2008/105/EC of the European Parliament and of the Council of 16 December 2008

6. European Parliament (2009) S.I. No. 272 of 2009 European Communities Environmental Objectives (Surface Waters) Regulations

7. Irish EPA (2006) Irish water framework directive monitoring programme V1.0, V1.0

8. ScorePP (2008) European initiatives: source control options for reducing emissions of priority pollutants, 2012

9. Clarke R, Roberts A, Conrad A (2009) Chemical prioritisation: ranking chemicals of concern to Scotland's environment, Phase 1: Surface Waters

10. Ahlman S, Svensson G (2005) SEWSYS - a tool for simulation of substance flows in urban sewer systems, ISSN 1650-3791; no 2005:11

11. De Keyser W, Gevaert V, Verdonck F, De Baets B, Benedetti L (2010) An emission time series generator for pollutant release modelling in urban areas. Environ Model Softw 25:554-561

12. Fehily B (2008) A review of and report on certain matters relating to Dublin city Council's Ringsend Wastewater Treatment Plant

13. National Roads Authority (2010) National roads authority report: national route lengths as of 31/12/2009

14. CSO (2009) Central Statistics Office Yearly Report

15. Seriki K, Gasperi J, Castillo L, Scholes L, Eriksson E, Revitt M et al (2008) Priority pollutants behaviour in end of pipe wastewater treatment plants. Deliverable Rep 5:1-91 